\title{
Design optimization of the satellite in precessional transmission
}

\author{
Stanislav Lealin* \\ Technical University of Moldova, Faculty of Mechanical Engineering and Transport, str. Studentilor, \\ 3/1, MD-2045, Chisinau, Republic of Moldova
}

\begin{abstract}
One of the important tasks in the design of gears and gearboxes based on them is to increase the load capacity, durability and noise reduction with moderate requirements for manufacturing precision by providing elastic compliance of the teeth with simple satellite manufacturing technology. In the paper is firstly presented a base satellite in a precessional transmission. The design is rigid and non-technological. There was simulated the workload and the results are presented as a linear deformation. Simulation was based on the special module for the calculation and analysis of complex structures using the integrated module Stress Analysis (program Autodesk Inventor Professional 2012). It uses the finite element method calculation, based on the ANSYS program. Then is shown an optimized satellite structure. This option provides an increased durability and noise reduction due to local and general tooth compliance. The main drawback of this satellite is the high laboriousness of making cut-outs in the satellite. In this satellite, by increasing the diameter of the radial channel, it is possible to reduce the wall thickness and thereby increase the compliance. The simulation results of a new satellite design are presented. Compliance increased roughly by 4 times compared to the base version. When the gear is in mesh with the satellite, due to the increased elastic compliance of the teeth, a multi-pairing mesh is achieved, which reduces the contact loads and noise, increases the service life of the transmission.
\end{abstract}

\section{Stress analysis. ANSYS. Theoretical calculation}

\subsection{Stress analysis. ANSYS}

The criteria used to determine the final configuration items for products are the durability and the rigidity. Unfortunately, the existing arithmetic formulas for finding the parameters of strength and stiffness are applicable only to simple circuit loading devices, in case when the item configuration is quite simple.

In more complex cases, the correct calculation of the strength and stiffness can be made only by the finite element method (Figure 1).

\footnotetext{
*Corresponding author: stanislav.lealin@gmail.com
} 


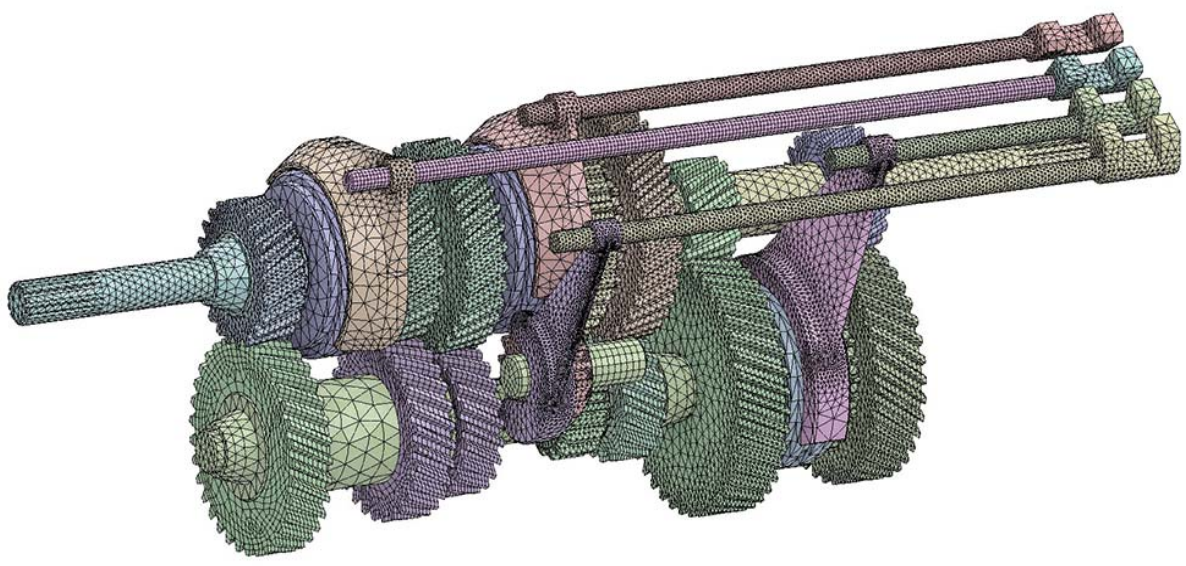

Fig. 1. Finite element example.

For these purposes, the Autodesk Inventor Professional [1] integrates a special module of ANSYS [2], which allows, for the existing geometry of the solid model parts, to quickly settle on the strength and stiffness. It is also possible to make a calculation of the strength of the parts while moving the mechanism; such a calculation is available after performing the dynamic analysis [3].

\subsection{Theoretical calculation}

Equivalent Stress. The equivalent stress (Figure 2) is calculated from the energy theory of strength by using the following formula (Eq. 1):

$$
\sigma_{e q}=\frac{1}{\sqrt{2}} \sqrt{\left(\sigma_{x}-\sigma_{y}\right)^{2}+\left(\sigma_{y}-\sigma_{z}\right)^{2}+\left(\sigma_{z}-\sigma_{x}\right)^{2}+6\left(\tau_{x y}^{2}+\tau_{z x}^{2}+\tau_{z y}^{2}\right)}
$$

$\sigma e q$ - stress equivalent uniaxial tension;

$\sigma x-\mathrm{x}$-axis normal stress;

$\sigma y-\mathrm{y}$-axis normal stress;

$\sigma z-\mathrm{z}$-axis normal stress;

$\tau_{x y}-\mathrm{xy}$-axis tangent stress;

$\tau_{z x}-\mathrm{zX}$-axis tangent stress;

$\tau_{z y}-$ zy-axis tangent stress;

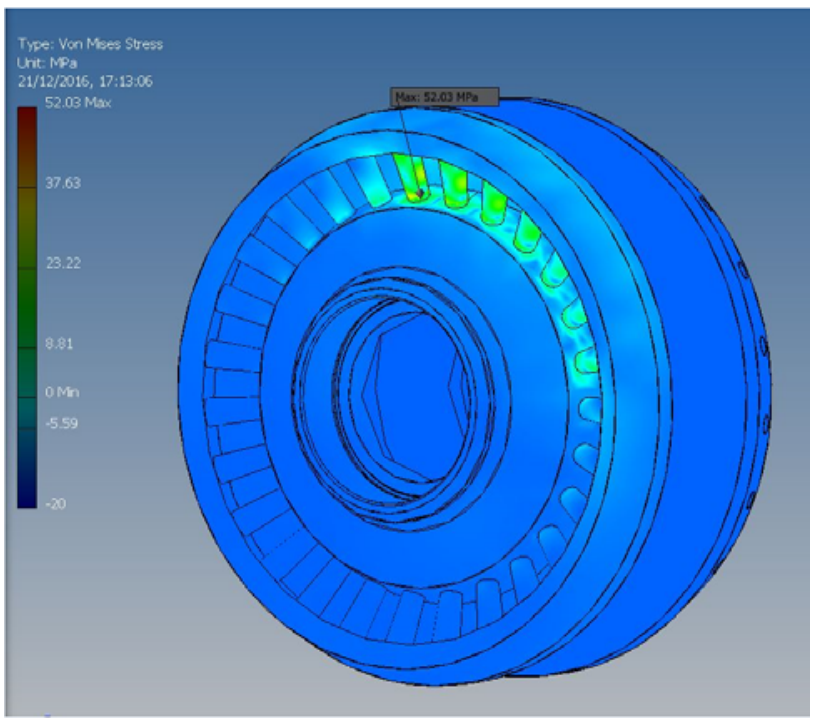

Fig. 2. Von Mises Stress, MPa. 
Reminding that in any volume element, there are both shear stresses and tensile stressstrain.

But if performing the calculation of all strains at a given point in the rotated coordinate system, then their value will be different. Thus, it is possible to achieve such a rotation of the coordinate system that the shear stresses are absent and the tensile stress-strain will be the maximum possible value. These extreme stresses are called principal stresses.

It is necessary to consider the strength of the material, if the criterion doesn't respond to the strength energy conditions, e.g. the brittle materials (glass, ceramics) have a compressive strength that is much higher than in tension.

The parameters of finite elements:

The construction of an ordered grid requires a preliminary model partitioning into separate components with simple geometry, and then selection of attributes of an element and the corresponding control commands quality mesh, so that a finite element model with an ordered grid can be built.

Created by ANSYS program [4], the ordered grid may consist of hexagonal, rectangular and triangular elements.

For the triangular grid, the program allocates areas of the model for applying an ordered grid, creates a first rectangular grid, and then turns it into a grid of triangular elements.

As described previously, the program works by the finite element method. If necessary, we can set the number of items manually, or let the program automatically split the model into a certain number of elements (Figure 3).

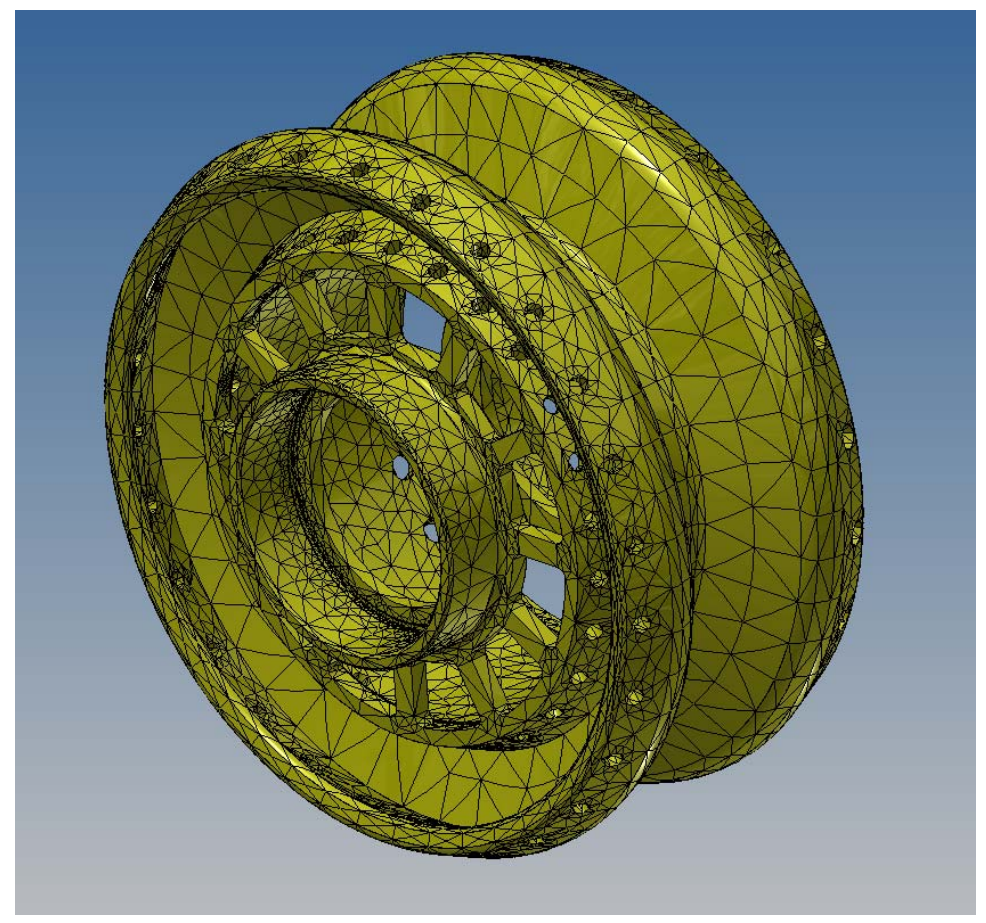

Fig. 3. Finite elements Mesh example. Elements: 56794 (default mesh configuration).

\section{Optimization of the satellite. Conclusion}

One of the important tasks in the design of gears and gearboxes based on them is to increase the load capacity, durability and noise reduction with moderate requirements for 
manufacturing precision by providing elastic compliance of the teeth with simple satellite manufacturing technology.

All loads were based on motor power, standard for these type of reducers, of $1 \mathrm{~kW}$.

In Figure 4 is presented a base satellite in a precessional transmission. The design is too rigid (linear deformation $=0.004442 \mathrm{~mm}$ ) and non-technological. In (Figure 4) the workload is simulated, the results are presented as a linear deformation.

In Figure 5 is shown an optimized satellite structure (linear deformation $=0.01606 \mathrm{~mm}$ ). Such a significant difference in linear deformation (almost 400\%) significantly increases the contact spot (the number of teeth that are simultaneously meshed) of the satellite rollers with the fixed gear teeth of the reducer.

This option provides an increased durability and noise reduction due to local and general tooth compliance.

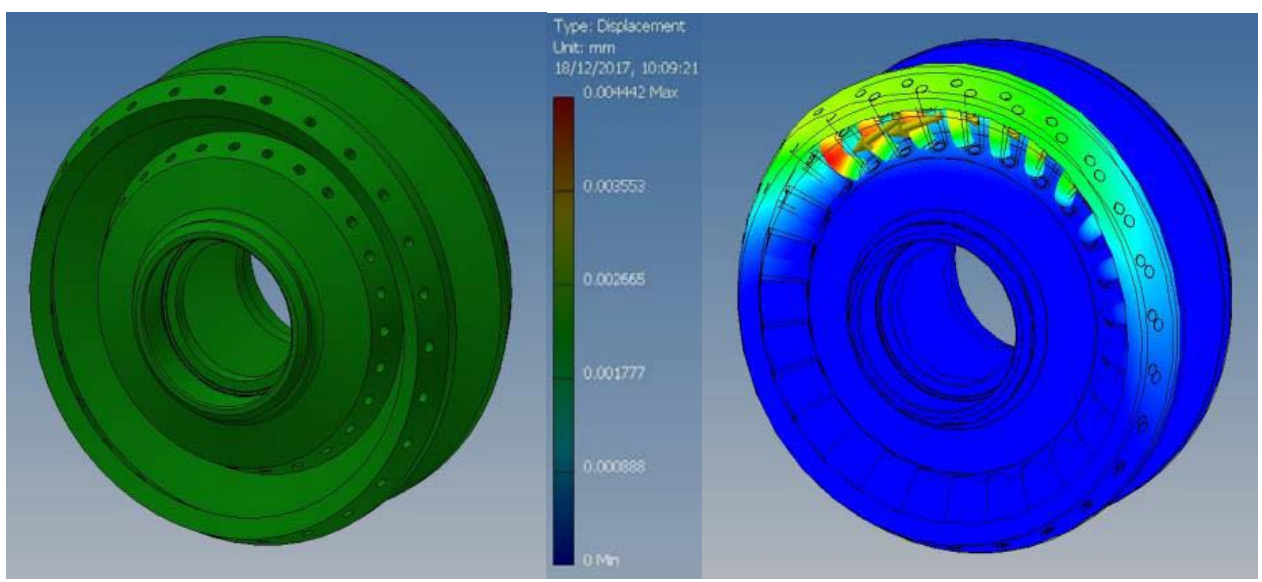

Fig. 4. Base satellite structure/ Linear deformation in $\mathrm{mm} /$ Simulation.

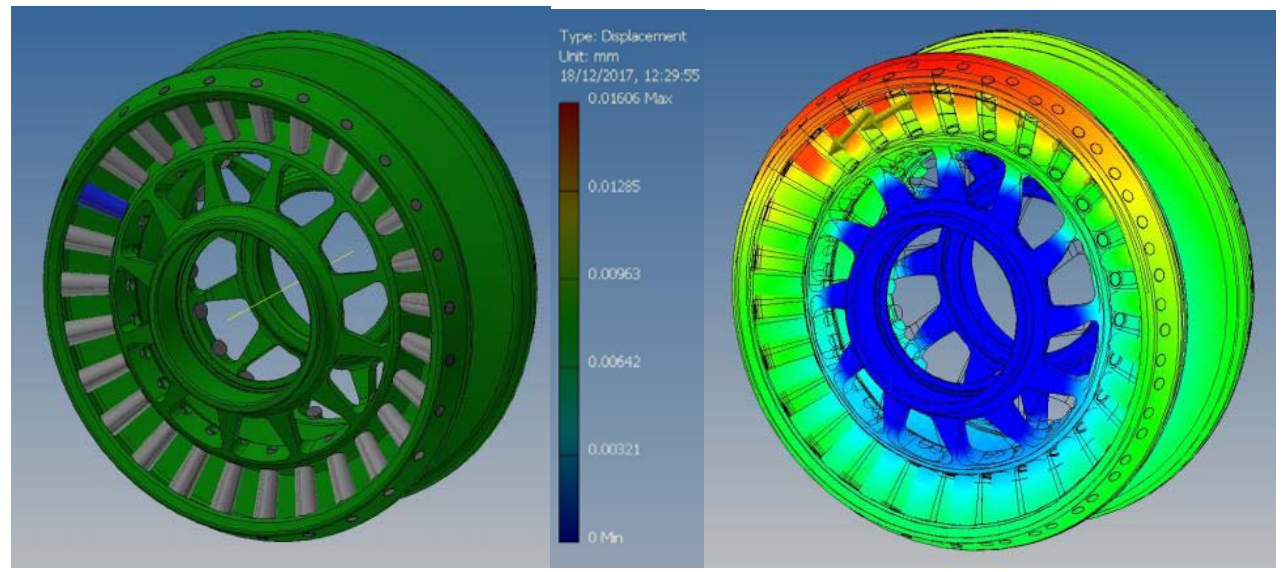

Fig. 5. Optimized satellite structure is shown/ Linear deformation in $\mathrm{mm} /$ Simulation.

The main drawback of this satellite is the high laboriousness of making cut-outs in the satellite. In this satellite, by increasing the diameter of the radial channel, it is possible to reduce the wall thickness (Figure 6) and thereby increase the compliance. 




Fig. 6. Base design in the red colour. Optimized design in the green colour.

All these improvements will reduce the mass and dimension parameters of the satellite, while increasing its flexibility not only on the teeth, but also on the rest of the satellite casing.

The presented design is optimal from a technological point of view.

When considering more thin-walled structures, compliance indicators were even better, but design firmness gets too low, which is unacceptable.

In Figure 5 there can be seen the simulation results of a new satellite design. Compliance increased roughly by 4 times compared to the base version.

When the gear is in mesh with the satellite, due to the increased elastic compliance of the teeth, a multi-pairing mesh is achieved, which reduces the contact loads and noise, increases the service life of the transmission.

\section{References}

1. W. Younis, Up and running with Autodesk Inventor Professional 2012: Part 1, Stress and Frame Analysis (Wasim Younis ed., 2012)

2. S. Lealin, Stress Analysis, Frame Analysis and Calculation of Metal Structures in Inventor Software, Applied Mechanics and Materials 809-810, 871-877 (2015)

3. A.B. Kaplun, E.M. Morozov, ANSYS - in the hands of the engineer (Mechanical Engineering Publishing House, Moscow, 2003)

4. How to use stress analysis in Autodesk Inventor to test your parts. Available at "http:/www.instructables.com/id/How-to-use-stress-analysis-in-Autodesk-Inventor-totest-your-parts/, accessed 20.01.2015 